\title{
A model to predict the collapsibility of Loess from micro-structural parameters
}

\author{
Gao lingxia1, a, Yang xiangjun ${ }^{2, b}$ and Li Shunqun ${ }^{3, c}$ \\ ${ }^{1}$ School of Civil Engineering, Dalian Nationalities University, Dalian, 116600, China \\ ${ }^{2}$ Municipal Facilities Construction Corporation, Dalian 116011, China \\ ${ }^{3}$ School of Civil Engineering, Tianjin Chengjian University, Tianjin 300384, China \\ agaolingxia2006@126.com, bxiangjunyang2003@163.com, clishunqun@sina.com
}

\begin{abstract}
Keywords: loess, micro-structural parameters, collapsibility, prediction model
Abstract. The collapsibility has become a key material property to study strength and deformation behavior of loess, which is closely related with its micro structural character. In this paper, the authors firstly obtained simple micro-structural parameters both on loess samples of before and after collapsing from the Electron Scanning Microscope images, then made a comparative analysis of relationship among these simple parameters and constructed synthetic micro-structural parameters. Finally the authors presented equations for prediction the relationship between collapsibility coefficient and synthetic micro-structural parameters. It is shown that the collapsibility of loess can be evaluated objectively using the proposed method. The study provides efficient exploration in quantificational studying the effects of microstructure on collapsibility of loess.
\end{abstract}

\section{Introduction}

Collapsibility is a large concern in foundation design that deals with loess-like subsoil, as differential collapse deformations in loess often result in mainly problems in engineering construction [1-3]. In order to ensure the engineering safety and to reduce the cost, it is necessary to reasonably evaluate collapsibility of loess based on the characteristics of loess and engineering requirement. The mechanics and properties of these natural deposits have been systematically investigated [4-8]. A large number of studies have shown that there is a direct correlation between the collapsibility of loess and its microstructure. Several empirical correlations have been developed to estimate collapsible property of loess [9-11]. However, any of these equations cannot introduce microstructure parameter into collapsibility evaluation.

In this paper, The Electron Scanning Microscope images were obtained both on loess samples of before and after collapsing, then corresponding micro-structural parameters were obtained. The relationship among these parameters was discussed and the synthetic micro-structural parameters were constructed as well. The calculation method of coefficient of collapse was established. The collapsibility of loess can be evaluated objectively using the proposed method. The efficient exploration was made in quantificational studying the effects of microstructure on collapsibility of loess.

\section{Sampling and laboratory test}

The studied loess was natural un-disturbance taken from $2 \mathrm{~m}$ depth exploratory wells on a terrace in $\mathrm{Xi}$ 'an city in order to obtain high-quality samples. The main mechanics indexes of the loess constitute of the following: natural density, $\rho=1.53 \mathrm{~g} / \mathrm{cm}^{3}$, specific gravity of the soil particles, $G_{\mathrm{s}}=2.72$, natural water content, $w=16.8 \%$. Titration method was used to preparation samples with different moisture content. The resulting samples were classified into 3 identical groups in terms of water content. After water and air contained in the samples arrived in the equilibrium condition due to capillary action and thermal force, the samples were consolidated in different pressure, when deformation under given pressure being stable, the samples were saturated and the collapsible test were carried out. The single line method was applied in collapsible test. The results are shown in following table 1. 
Table 1. Results of collapsible tests of samples with different water content

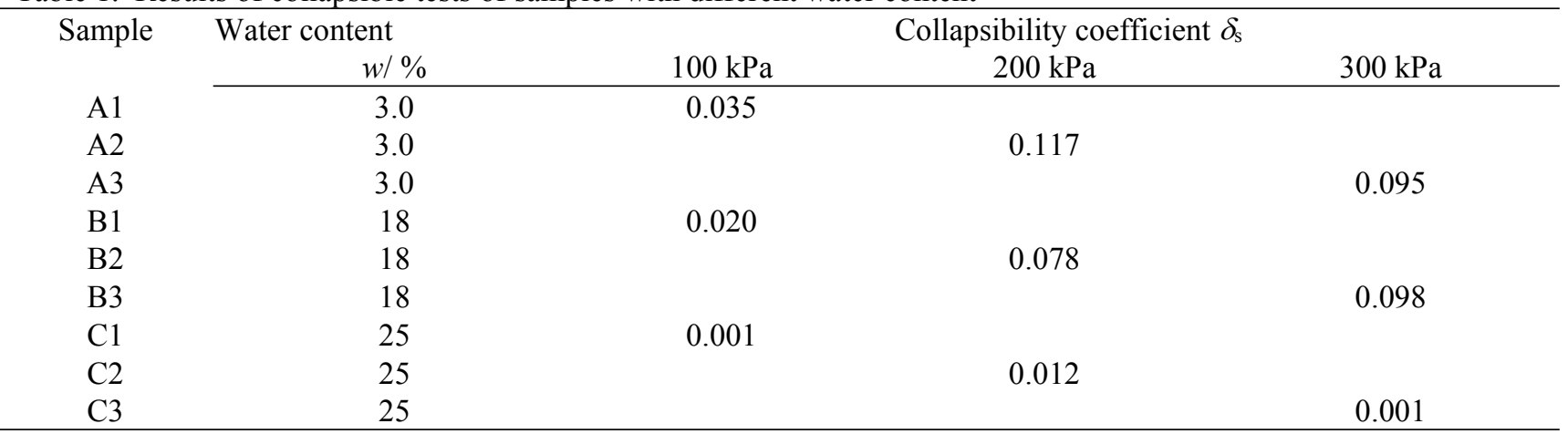

\section{Micro-structural Parameters Extraction and Construction}

For the sake of comparison, the microstructure samples before and after collapsing were prepared using frozen dried method respectively, and then scanning electron microscope (SEM) tests were carried out. SEM images were obtained and micro-structural parameters were extracted as well. The approach can be refered to the paper written by Gao lingxia[12].

Figure 1 shows SEM photos of before and after collapsing loess with water content $25 \%$ under consolidation pressure 200 and $300 \mathrm{kPa}$. The right is photo of before collapsing. From Figure 1, it can be seen that after collapsing, the space occupied by soil particle increases significantly, on the contrary, the space occupied by soil pore decreases obviously. The image characteristics are in conformity with the inner mechanism of loess collapsibility.

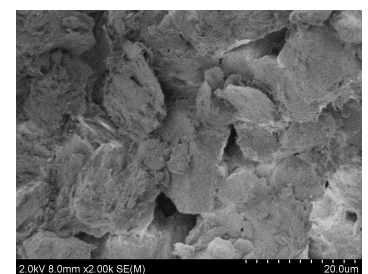

Before collapse

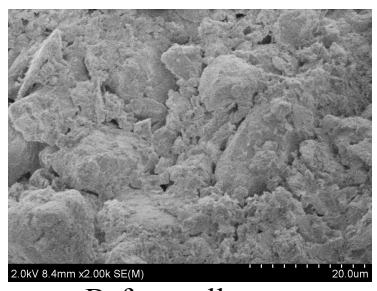

Before collapse

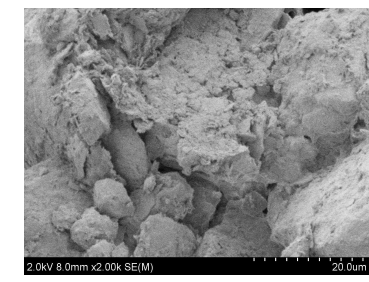

After collapse

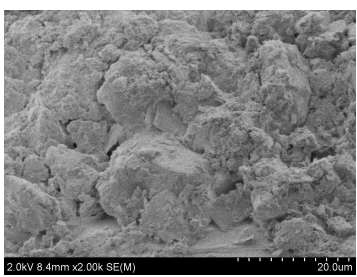

After collapse

Figure. 1 Comparison of SEM images between sample before and after collapsing under different conditions $(w=25 \%)$

Simple Microstructure Parameters Obtaining from SEM Images. The Leica QWin software was employed to pick up micro-structure parameters, and the obtained micro-structure parameters can be written as follow matrix

$$
x=\left(x_{1}, x_{2}, x_{3}, x_{4}, x_{5}, x_{6}, x_{7}, x_{8}, x_{9}\right)^{\mathrm{T}}
$$

Here, $x_{1}$ and $x_{2}$ are respectively the sum of horizontal intercept and the sum of vertical intercept of section of pore in soil particle, $x_{3}$ and $x_{4}$ are the sum of the circumference of every pore and the total number of pores, $x_{5}$ is the area ratio of the total pore to field area, $x_{6}$ is coefficient of anisotropy, $x_{7}$ is filling ratio, $x_{8}$ is average chord. And $x_{9}$ is a grey threshold determined by the Leica QWin software when the maximal variance achieved. It is clear that the primary properties of the pore micro-structure in the analysis field, including size, shape, gradation, anisotropy and so on, can be characterized by the stated parameters above .

Different parameters of microstructure pictures from different SEM photo under the same condition were merged and they average were calculated, respectively. As analyzing the 
collapsibility loess under different states before and after the microstructure characteristic, mean value of microstructure parameters were applied.

Synthetic Micro-structural Parameters. According to studies on loess SEM images[13], principle component index of microstructure parameters can be used to establish the constitutive relationship to evaluate the collapsible deformation behavior of loess, which can reflect the inherent relation between microstructure and macro engineering properties, thus principle component analysis is introduced to construct synthesis parameters.

Based on principal component analysis method[14], the corresponding characteristic value of the standardized microstructure parameters $x_{i}(i=1 \sim 9)$, their proportion and cumulative contribution rate are shown in Table 2.

Table 2 Eigenvalues and corresponding principal component contribution

\begin{tabular}{|c|c|c|c|c|}
\hline No. & Eigenvalues & margins & contribution & $\begin{array}{l}\text { cumulative } \\
\text { contribution }\end{array}$ \\
\hline & $\lambda_{k}$ & & $\eta_{i} / \%$ & $\xi_{i} / \%$ \\
\hline 1 & 5.021 & 2.078 & 55.79 & 55.79 \\
\hline 2 & 2.943 & 2.214 & 32.70 & 88.49 \\
\hline 3 & 0.729 & 0.473 & 8.10 & 96.59 \\
\hline 4 & 0.256 & 0.225 & 2.84 & 99.43 \\
\hline 5 & 0.031 & 0.015 & 0.35 & 99.77 \\
\hline 6 & 0.016 & 0.012 & 0.18 & 99.95 \\
\hline 7 & 0.004 & 0.003 & 0.04 & 99.99 \\
\hline 8 & 0.001 & 0.001 & 0.01 & 100.00 \\
\hline 9 & 0.000 & & 0.00 & 100.00 \\
\hline
\end{tabular}

The corresponding eigenvectors also can be obtained, according to the corresponding eigenvectors, 9 principal component $p_{i}(i=1 \sim 9)$ expressions can be obtained. For example, the first principal component is a linear combination of vector $p_{1}$ and the standardized value of microstructure characteristics.

According to principal component expression can be concluded that the principal component scores of each sample, and then the samples according to the principal component scores can be based on the evaluation of the microstructure theory.

\section{Relation between Collapsibility and Microstructure Character}

Principal Component Scores. The three principal components can be used to express SEM microstructure information after standardized. The corresponding cumulative contribution rate can be up to $97 \%$. The three principal components (PC) are as following, respectively.

$$
\begin{gathered}
\boldsymbol{P}_{1}=0.283 x_{1}+0.380 x_{2}+0.380 x_{3}+0.376 x_{4}-0.324 x_{5}-0.158 x_{6}-0.352 x_{7}-0.421 x_{8}+0.239 x_{9} \\
\boldsymbol{P}_{2}=0.446 x_{1}+0.232 x_{2}+0.281 x_{3}+0.128 x_{4}+0.385 x_{5}+0.383 x_{6}+0.337 x_{7}-0.117 x_{8}-0.480 x_{9} \\
\boldsymbol{P}_{3}=0.072 x_{1}-0.394 x_{2}-0.227 x_{3}+0.264 x_{4}-0.211 x_{5}+0.767 x_{6}-0.190 x_{7}-0.152 x_{8}+0.161 x_{9}
\end{gathered}
$$

Table 3 shows Scores of the first, the second and the third principal components for the before collapsing samples and scores of cumulative principal component.

Relationship between Collapsibility Coefficient and Cumulative Principle Component. From Table 3, we can know that the specimens $\mathrm{C} 1, \mathrm{C} 2$ and $\mathrm{C} 3$ are refer to nocollapsible loess, B1 corresponds to loess with light collapsibility, A1 corresponds to loess with medium collapsibility, other states represent strong loess collapsibility. 
$\underline{\text { Table } 3 \text { Scores of principal components for the before collapsing samples and comparative analysis of their collapsibility }}$

\begin{tabular}{|c|c|c|c|c|c|c|}
\hline \multirow{2}{*}{$\begin{array}{l}\text { initial } \\
\text { state }\end{array}$} & \multirow{2}{*}{$\boldsymbol{P}_{1}$} & \multirow{2}{*}{$\boldsymbol{P}_{2}$} & \multirow{2}{*}{$\boldsymbol{P}_{3}$} & \multirow[t]{2}{*}{$P$} & $\begin{array}{c}\text { collapsibility } \\
\text { coefficient }\end{array}$ & \multirow[t]{2}{*}{ collapsible } \\
\hline & & & & & $\delta_{\mathrm{s}}$ & \\
\hline A1 & 1.82 & 1.02 & 0.33 & 3.17 & 0.035 & ys \\
\hline $\mathrm{A} 2$ & 4.24 & 2.83 & 1.21 & 8.29 & 0.095 & $y$ \\
\hline A3 & 7.23 & 3.24 & 2.30 & 12.78 & 0.117 & \\
\hline B1 & 1.65 & 0.86 & 0.21 & 2.73 & 0.020 & \\
\hline $\mathrm{B} 2$ & 3.65 & 2.02 & 1.04 & 6.71 & 0.078 & \\
\hline B3 & 3.99 & 2.23 & 1.26 & 7.48 & 0.098 & \\
\hline $\mathrm{C} 1$ & 1.22 & 0.88 & 0.32 & 2.41 & 0.001 & \\
\hline $\mathrm{C} 2$ & 1.54 & 0.68 & 0.37 & 2.58 & 0.012 & \\
\hline $\mathrm{C} 3$ & 1.14 & 0.35 & 0.23 & 1.73 & 0.001 & $n$ \\
\hline
\end{tabular}

In fact, the forging loess specimens in various states are derived from a collapsible loess, the difference of collapsibility judged result from their various hydraulic conditions. With the increasing of the initial moisture content, the loess collapsibility reduces gradually, with the increasing of pressure, the loess collapsibility increases gradually. Therefore, to some extent, the collapsibility of loess is not only depends on its initial state, but also depends on the initial moisture content and its corresponding stress conditions. In order to study the relation between collapsibility of loess and the corresponding microstructure parameters, the relation between the 6 kinds of collapsible sate which representing coefficient of collapsibility is greater than or equal to 0.015 and their accumulated principal component were dotted in rectangular coordinate system, which is shown in Figure 2.

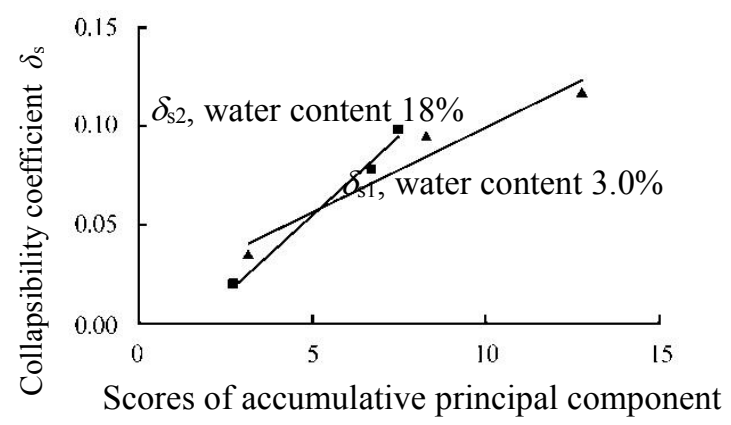

Figure 2 Relationships between collapsibility coefficient and scores of accumulative principal component.

According to Figure 2, linear statistical relationships between coefficient of collapsibility and cumulative principal component were obtained, that is

$$
\begin{aligned}
& \delta_{\mathrm{s} 1}=0.0086 p+0.0128 \\
& \delta_{\mathrm{s} 2}=0.0158 p-0.0237
\end{aligned}
$$

Thus, it is can be known that there is linear relation between coefficient of collapsibility of and its cumulative principal component. Collapsibility of loess can be predicted according to the principal component. Firstly, obtain the photo of loess under certain pressure based on SEM, then obtain the microstructure parameters applied image processing software and principle component used introduced method in this paper, at this point you can according to the equation (5)and equation (6) predict collapsibility coefficient corresponding to different moisture content. When moisture content is close to $3.0 \%$, the line $\delta_{\mathrm{s} 1}$ is used, when moisture content is close to $17 \%$, the line $\delta_{\mathrm{s} 2}$ is used and for other moisture content prediction can adopt the method of interpolation.

Conclusions should state concisely the most important propositions of the paper as well as the author's views of the practical implications of the results.

\section{Conclusions}

Loess collapsibility depends on not only the sample initial state, but also its initial moisture content and the corresponding consolidation stress conditions. This paper introduces the approaches of 
principal component analysis to study characteristics of loess microstructure and constructed principle component method to predict the collapsibility of loess. According to SEM photo, the original microstructure parameters were extracted, based on principle component analyzing, the first, the second and third principal components were calculated as well as scores of principal components, whose accumulative contribution is $96 \%$, can exhibit almost all information expressed by the nine original microstructure parameters. The results show that with the increase of consolidation pressure, the first principal component increase by approximate linear, the loess collapsibility coefficient has a linear relation with its accumulated principal component. According to this important understanding, the collapsibility coefficient calculation method is established based on principal component scores, and collapsibility loess evaluation method is established.

Samples due to the microstructure and SEM photos carry large amounts of information, if the number of test point increase, there must exist growth rapid of microcosmic test scale and huge difficulties in data processing. As an exploratory study, this paper only select three test points, therefore, this study is preliminary. Develop greater amounts of collapsibility loess research and the corresponding microscopic mechanism will be the next focus on the direction of the effort.

\section{Acknowledgements}

This work was financially supported by China National Science Foundation (51178290), the Fundamental Research Funds for the Central Universities of Grant (DC201502040402) and by the Ministry of Construction Project (k4201252). In addition, the authors would like to thank the reviewers and the associate editor for their valuable comments, which have improved the quality of this paper.

\section{References}

[1] Gibbs H.J.\& Holland W.Y.. In: United States Department of the Interior Bureau of Reclamation, Engineering Monograph 28,(1960), p.1-37

[2] Rogers C.D.F. , Dijkstra T.A., Smalley, I.J. Engineering Geology Vol.37, No.2 (1994), p. 83-113

[3] Leroueil S. \& Vaughan P.R. Geotechnique Vol.40, No.3, (1990), p.467-488

[4] Assallay, A.M. Rogers C.D.F., Smalley I.J.. Journal of Arid Environments, Vol.32, No.4 (1996), p. $373-386$

[5] Luo, Y.S.. Chinese Journal of Geotechnical Engineering Vol. 20, No.4 (1998), p.87-91

[6] Nouaouria M.S., Jefferson I.F. Mavlyanova N. , Hara-Dhand K. O. Engineering Geology Vol.99 (2008), p. 85-90

[7] Hounston S. L. Houston W.N. , Zapata C.E.. Geotechnical and Geological Engineering Vol. 19, NO. 34 (2001), 9.333-355

[8] Gallipoli, D. Gens, R. Sharma, J. Vaunat.. Geotenique Vol. 53, No. 1(2003), p. 123-135

[9] Sun Q., Zhang X.K., Li H.E.. Chinese Rock and Soil Mechanics, Vol.29, No.8), (2008), p. 664672

[10] Liu H.S., Ni W.K., Yan B.. Rock and Soil Mechanics,Vol 29, No.8, (2008), p. 722-726

[11] Chen C.L., Gao P., He J.F.. Chinese Journal of Geotechnical Engineering, Vol. 29, No. 9, (2007), p.1330-1339

[12] Gao L.X. Miao. T. L., LI S.Q.., Journal of Convergence Information Technology, Vol.6, No.5(2011),p. 32-42

[13] Gao L.X., Miao T.L. Yang Q.. Chinese Rock and Soil Mechanics, Vol. 33, No.7, (2012), P.1921-1926.

[14] Johnson D. In: Applied Multivariate Methods for Data Analysts.Higher Education Press. (2006). 